\title{
Certain hereditary properties and metrizability in generalized ordered spaces
}

\section{by}

\author{
H. R. Bennett and D. J. Lutzer (Lubbock, Tex.)
}

Abstract. Using Bennett's theorem that a quasi-developable paracompact $p$-space must be metrizable we prove that any generalized ordered space whose every subspace is a $p$-space must be metrizable. In addition we sharpen a recent theorem of van Wouwe by showing that, in a generalized ordered space, Morita's $M$-spaces, Borges' $w / \mathcal{A}$-spaces and Creede's quasi-complete spaces are mutually equivalent notions.

1. Introduction. A completely regular space $X$ is a $p$-space if there is a sequence $\langle\mathscr{G}(n)\rangle$ of collections of open subsets of $\beta X$ equivalently, of any compactification of $X$, each covering $X$, and having the property that for every $x \in X$, $\bigcap\{\operatorname{St}(x, \mathscr{G}(n)) \mid n \geqslant 1\} \subset X$. (As usual, $\operatorname{St}(x, \mathscr{G}(n))$ denotes $\bigcup\{G \in \mathscr{G}(n) \mid x \in G\}$.) The sequence $\langle\mathscr{G}(n)\rangle$ is called a pluming of $X$ in the compactification. Spaces of this type were introduced in [1] as part of the characterization of perfect pre-images of metric spaces; more recently they have played a role in other parts of topology, perhaps most notably in metrization theory.

Several recent papers have involved hereditary $p$-spaces, i.e., spaces whose every subspace is a $p$-space [2]. The elegance of the results obtained amply justifies asking the question, "Which $p$-spaces are hereditarily $p$-spaces?" A moment's reflection shows that any $p$-space with a $G_{\delta^{-}}$-diagonal is hereditarily a $p$-space, but beyond that easy observation, conditions which are sufficient to force a space to be hereditarily a $p$-space are hard to find. In this paper we study generalized ordered ' spaces which are hereditarily $p$-spaces.

Recall that a generalized ordered space (GO space) is a $T_{1}$-space $(X, \mathscr{T})$ equipped with a linear ordering such that there is a base of open sets for $\mathscr{T}$ whose every member with a linear ordering suces are precisely those spaces which can be topologically embedded as subspaces of linearly ordered topological spaces (LOTS), i. e., linearly ordered sets equipped with the usual open interval topology.

\footnotetext{
Our paper is organized as follows. In Section 2 we sharpen a recent result due
}

(1) A subset $C$ is order-convex (in $(X,<)$ ) if $\{x \in X \mid a \leqslant x \leqslant b\} \subset C$ whenever $a, b \in C$. 
to van Wouwe, proving that in the class of GO spaces, Morita's $M$-spaces, Borges' $w \Delta$-spaces and Creede's quasi-complete spaces are mutually equivalent notions. (See Section 2 for relevant definitions and references. Van Wouwe's theorem estab. lished the equivalence of $M$-spaces and $w \Delta$-spaces in the class of GO spaces.) In Section 3 we show that any GO space which is hereditarily a $\beta$-space in the sense of Hodel must be paracompact. (The definition of a $\beta$-space is reproduced in Section 3.) Finally, in Section 4 we use the results of Sections 2 and 3 to prove our main theorem which asserts in part that any $\mathrm{GO}$ space $X$ which is hereditarily a $p$-space must be quasi-developable in the sense of Bennett [6] and therefore must be metrizable because, as proved in [5], any quasimdevelopable paracompact $p$-space must be metrizable.

Let us establish some terminology and notation. When considering GO spaces our usage will follow that of [12]; in particular, given points $x, y$ in a linearly ordered set $X$ we write $] x, \rightarrow[$ for $\{z \in X \mid x<z\}$ and $[x, y[$ for $\{z \in X \mid x \leqslant z<y\}$. A cardinal is an initial ordinal. The cardinality of a set $S$ is written $|S|$. We identify an ordinal with the set of its predecessors. Thus $\omega_{1}$ is identified with $\left[0, \omega_{1}\right.$ [ where $\omega_{1}$ is the first uncountable ordinal and $\omega_{0}$ is identified with the set $\left[0, \omega_{0}\right.$ [ consisting of all non-negative integers. For any cardinal $x$, the first cardinal greater than $x$ is denoted by $\chi^{+}$. Let $N=\left[1, \omega_{0}[\right.$.

In Section 4 it will be necessary to analyze the pseudogap structure of a GO space. Because the terminology to be used is not entirely standard we pause here to record the relevant definitions. Suppose $X$ is a GO space when equipped with the toplogy $\mathscr{T}$ and the linear ordering <. By a gap of $X$ we mean a pair $(A, B)$ of convex subsets covering $X$ such that if $a \in A$ and $b \in B$ then $a<b$ and such that the set $A$ has no supremum in $X$ and $B$ has no infimum in $X$. Thus gaps are simply Dedekind cuts in the linearly ordered set $(X,<)$. By a pseudogap of $X$ we mean a pair $(C, D)$ of nonvoid convex subsets of $X$ such that

1) $C \cup D=X$

2) if $c \in C$ and $d \in D$ then $c<d$,

3) $C$ and $D$ are $\mathscr{T}$-open sets,

4) either $C$ has a supremum $p$ in $X$ while $D$ has no infimum in $X$ (in which case $p$ is said to determine a right pseudogap of $X$ ) or else $C$ has no supremum in $X$ while $D$ has an infimum $q$ in $X$ (in which case $q$ is said to determine a left pseudlogup of $X$ ).

In less technical terms, $p$ determines a left pseudogap of $X$ if the set $[p, \rightarrow[$ is $\mathscr{T}$-open even though $p$ has no immediate predecessor in the ordering of $X$. For example, each point of the Sorgenfrey line determines a left pseudogap while each irrational in the Michael line determines both a left and a right pseudogap.

It is known $([12], 2.9)$ that there is a cannonical way to embed a GO space $X$ into a compact LOTS $X^{\text {\#k }}$ in such a way that the ordering of $X^{\#}$ extends the ordering of $X$. Indeed, whenever $Y$ is a compact LOTS which contains $X$ and whose order extends the ordering of $X$ (in which case $Y$ is called an ordered compactification of $X$ ) it is clear that if $p$ determines a left pseudogap of $X$, then there must be a point $p^{-}$ which is an immediate predecessor of $p$ in the ordering of $Y$ and which lies in $Y-X$. Analogous assertions hold for right pseudogaps of $X$ : each must have an immediate successor in any ordered compactilication of $\lambda^{\prime}$.

2. An extension of van Wouwe's theorem. Recall that Morita's $M$-spaces may be haracterized as those Hausdorff spaces which can be mapped onto a metric space characterized as those Hausdorff spaces which can be mapped onto a metric space bes, the $1 A$-spaces, where a space $X$ is a $w \Delta$-space if there is a sequence $\langle\mathscr{G}(n)\rangle$ of open covers of $X$ such that if $p \in X$ and if $x_{n} \in \operatorname{St}(p, \mathscr{G}(n))$ for each $n \geqslant 1$, then the secquence $\langle x(n)\rangle$ must cluster in $X$. Van Wouwe [16] proved

2.1. Throrem. The following properties of a GO space $X$ are equivalent:

a) $X$ is an $M$-spacis;

b) $X$ is a w $\Delta$-space.

In [8] Creede introduced the notion of a quasi-complete space to aid in his study of metrization theory. A space $X$ is quasi-complete if there is a sequence $\langle\mathscr{H}(n)\rangle$ of open covers of $X$ such that if $A_{0} \supset A_{1} \supset \ldots$ is a decreasing sequence of nonempty closed sets such that for some $p \in X$ each $\mathscr{H}(n)$ contains a set $H_{n}$ with $\{p\} \cup A_{n} \subset H_{n}$, then $\cap\left\{A_{n} \mid \dot{n} \geqslant 1\right\} \neq \varnothing$. Such an $\langle\mathscr{H}(n)\rangle$ is called a quasi-complete sequence for $X$. Our first lemma gives an easy translation of that definition.

2.2. Lemma. A regular space $X$ is quasi-complete if and only if there is a sequence $\langle\mathscr{H}(n)\rangle$ of open covers of $X$ such that if $p \in X$ and if a sequence $\langle x(n)\rangle$ of points of $X$ has the property that for every $n$ some member of $\mathscr{H}(n)$ contains $\{p\} \cup\{x(k) \mid k \geqslant n\}$, then the sequence $\langle x(k)\rangle$ must cluster in $X$.

We can now give an extension of van Wouwe's theorem, partially answering a question of Creede [8].

2.3. THEOREM. Let $X$ a GO space. Then the following are equivalent:

a) $X$ is an $M$-space;

b) $X$ is a wd-space;

c) $X$ is quasi-complete.

Proof. The equivalence of a) and b) is Theorem 2.1 and b) obviously implies c). We show c) implies b). Let $\langle\mathscr{H}(n)\rangle$ be a sequence of open covers of $X$ as described in 22 ; members of each $\mathscr{H}(n)$ are order convex and that $\mathscr{H}(n+1)$ refines $\mathscr{H}(n)$. Let us say that a point $p$ is right-improper if there is and that $\mathscr{H}(n+1)$ refines $\mathscr{H}(n)$. Let us say that a point $p$ is right-improper if the a sequence $x(1)<x(2)<\ldots$ of points of $X$ such that $x(n) \in \operatorname{St}(p, \mathscr{H}(n))$ for each $n$ and yet the supremum of the sequence is a gap (or left-pseudogap) of $X$. Points which are left-improper are analogously defined, using decreasing sequences. Let $R$ be whe set of left-improper points of $X$.

There are two main steps in the proof. The second is the left-hand analogue f the first which we describe in some detail. We will construct open covers $\left\langle\mathscr{H}^{\prime}(n)\right\rangle$ of the first which we describe in some detail. We where $p \in X-L$ then $\langle y(n)\rangle$ clusters in $X$. The second step of the proof would analogously 
construct open covers $\mathscr{H}^{\prime \prime}(n)$ of $X$ which refine the covers $\mathscr{H}^{\prime}(n)$ and which would be $w \Delta$-sequences even at points of $L$. But then the covers $\mathscr{H}^{\prime \prime}(n)$ would constitute a $w \Delta$-sequence at all points of $X$, as required. We now turn to the first step of the proof.

\section{Consider a fixed $p \in R$. Then:}

(1) If $\langle x(n)\rangle$ and $\left\langle x^{\prime}(n)\right\rangle$ are two increasing sequences of points such that $\left\{x(n), x^{\prime}(n)\right\} \subset \operatorname{St}(p, \mathscr{H}(n))$ and such that $\sup \{x(n) \mid n \geqslant 1\}=v$ and $\sup \left\{x^{\prime}(n) \mid n \geqslant 1\right\}=v^{\prime}$ where both $v$ and $v^{\prime}$ are gaps or left-pseudogaps of $X$, then $v=v^{\prime}$.

(2) Let $v_{p}$ denote the unique gap or left-pseudogap whose existence is guaranteed by (1) and the definition of $R$. Then $\left[p, v_{p}[\subset \operatorname{St}(p, \mathscr{H}(n))\right.$ for every $n \geqslant 1$. Furthermore, if $p<x<v_{p}$ then the set $[p, x]$ is a countably compact subspace of $X$ because $\langle\mathscr{H}(n)\rangle$ is a quasi-complete sequence for $X$.

(3) With $v_{p}$ as in (2) there is some $n \geqslant 1$ such that $\left.\operatorname{St}(p, \mathscr{H}(n)) \subset\right] \leftarrow, v_{p}[$. For otherwise the sequence $\langle x(n)\rangle$, whose existence is guaranteed by the definition of $R$, would have to cluster in $X$ because $\left\langle\mathscr{H}^{\prime}(n)\right\rangle$ is a quasi-complete sequence of covers for $X$.

(4) For each $p \in R$ let $n(p)$ be the first integer $k$ having $\operatorname{St}(p, \mathscr{C}(k)) \subseteq] \leftarrow, v_{p}[$. Let $R(k)=\{p \in R \mid n(p)=k\}$. Define $J(k, p)=\bigcup\left\{\left[q, v_{q}[\mid q \in R(k)\right.\right.$ and $\left.v_{q}=v_{p}\right\}$. Let $\mathscr{J}(k)=\{J(k, p) \mid p \in R(k)\}$. Then each $\mathscr{J}(k)$ is a discrete collection of convex subsets of $X$ because for each $x \in X$ the open set $\operatorname{St}(x, \mathscr{H}(k))$ can meet at most two distinct members of $\mathscr{J}(k)$. And if $J(k, p)$ and $J(k, q)$ are distinct members of $\mathscr{J}(k)$ meeting $\mathrm{St}(x, \mathscr{H}(k))$ then either: $\operatorname{St}(x, \mathscr{H}(k))-J(k, p)$ or $\operatorname{St}(x, \mathscr{H}(k))-J(k, q)$ is a neighborhood of $x$ meeting only one member of $\mathscr{J}(k)$

(5) For each $J \in \mathscr{J}(k)$ choose one $p_{J} \in R$ such that $J=J\left(k, p_{J}\right)$ and then choose a strictly increasing sequence $\langle x(J, n)\rangle$ of points of $J$ having $p<x(J, 1)$ $<x(J, 2)<\ldots$ and which is cofinal in $J$. Let $D(k)=\{x(J, n) \mid J \in \mathscr{H}(k), n \geqslant 1\}$. Then $D(k)$ is a closed discrete subspace of $X$.

(6) Because any GO space is collectionwise normal [14], for each $k$ there is a pairwise disjoint collection $\mathscr{E}(k)=\left\{E(k, x) \mid x \in \bigcup_{j=1}^{k} D(j)\right\}$ of open convex subsets of $X$ such that

(a) $x \in E(k, x)$ whenever $x \in \bigcup_{j=1}^{k} D(j)$;

(b) $\mathscr{E}(k)$ refines the cover $\mathscr{H}(k)$.

(7) For each $H \in \mathscr{H}(k)$ define a collection $\mathscr{C}(H, k)=\{I I I$ is a convex component of the set $\left.H-\bigcup_{j=1}^{k} D(j)\right\}$. Then define

$$
\mathscr{H}^{\prime}(k)=\mathscr{E}(k) \cup(\bigcup\{\mathscr{C}(H, k) \mid H \in \mathscr{H}(k)\}) .
$$

Each $\mathscr{H}^{\prime}(k)$ is an open cover of $X$ and $\mathscr{H}^{\prime}(k+1)$ refines both $\mathscr{H}(k+1)$ and $\mathscr{H}^{\prime}(k)$.

We now prove that $\left\langle\mathscr{H}^{\prime}(k)\right\rangle$ acts as a $w \Delta$ sequence at all points of $X-L$. Fix $p \in X-L$ and suppose $y(n) \in \mathrm{St}\left(p, \mathscr{H}^{\prime}(n)\right)$. The sequence $\langle y(n)\rangle$ has a monotonic subsequence; without loss of generality we may assume $\langle y(n)\rangle$ is itself monotonic. Consider the case where $y(n)<y(n+1)$. If $y(n)<p$ for each $n$, then $\langle y(n)\rangle$ must cluster because $\langle\mathscr{H}(n)\rangle$, and hence $\left\langle\mathscr{H}^{\prime}(n)\right\rangle$, is a quasi-complete sequence for $X$. So suppose $y(n)>p$ for each $n \geqslant N_{0}$. If $p \notin R$ then $\langle y(n)\rangle$ must cluster in $X$, so assume $p \in R$. Find $k$ such that $p \in R(k)$. Then $J(k, p) \in \mathscr{J}(k)$; for notational simplicity write $J_{0}=J(k, p)$. Then there is a $p_{0} \in D(k)$ with $J_{0}=J\left(k, p_{0}\right)$ (see (5)). Let $n_{0}$ be the first integer such that $x\left(J_{0}, n_{0}\right)>p(\operatorname{see}(5))$. Then St $\left.\left.\left(p, \mathscr{H}^{\prime}(k)\right) \subset\right] \leftarrow, x\left(J_{0}, N_{0}+1\right)\right]$ so that for $n \geqslant N_{0}, y(n)$ belongs to the set $\left[p, x\left(J_{0}, n_{0}+1\right)\right]$ which is countably compact (see (2)). Hence $\langle y(n)\rangle$ clusters in $X$. Finally consider the case where $y(n)>y(n+1)$ for each $n$. If $y(n) \geqslant p$ for each $n$, then $\langle y(n)\rangle$ must cluster because $\langle\mathscr{H}(n)\rangle$, and therefore $\left\langle\mathscr{H}^{\prime}(n)\right\rangle$, is a quasi-complete sequence for $X$. Hence assume $y(n)<p$ for each $n \geqslant N_{1}$. If $\langle y(n)\rangle$ does not cluster in $X$, then $p \in L$ contrary to our choice of $p$. This completes the first step of the proof, and the second step is analogous, as explained above.

We now describe an easy example to which later sections refer. Its properties are easily verified.

2.4. EXAMPLE. Let $X=\left(\left[0, \omega_{1}\right) \times \omega_{0}^{*}\right) \cup\left\{\left(\omega_{1}, 0\right)\right\}$ be ordered lexicographically, where $\omega_{0}^{*}$ denotes the set $\omega_{0}$ with the reverse ordering (i. e., the set of negative integers). With the usual open interval topology of that ordering, $X$ is not first countable even though $X$ is hereditarily a $\beta$-space [11] and is the union of two metrizable subspaces, viz., $X-\left\{\left(\omega_{1}, 0\right)\right\}$ and $\left\{\left(\omega_{1}, 0\right)\right\}$. Furthermore, no increasing sequence in $X-\left\{\left(\omega_{1}, 0\right)\right\}$ can converge, so $X$ is not quasi-complete.

The following diagram summarizes the relationships among the various properties considered in this section. Notations near arrows refer to results in this paper or in another reference which establish the indicated arrows.

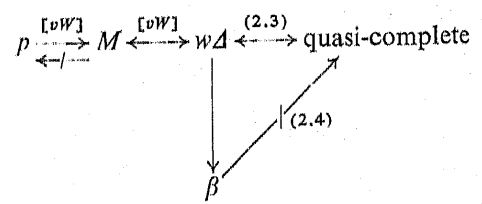

3. Hereditary properties and paracompactness in GO spaces. Recall that a topological space $(X, \mathscr{T})$ is a $\beta$-space [11] if there is a function $B: N \times X \rightarrow \mathscr{T}$, called a $\beta$-finction for $X$, such that:

(a) for each $x \in X$ and $n \geqslant 1, B(n, x)$ is a neighborhood of $x$; and

(b) if $\left\langle x_{n}\right\rangle$ is a sequence of points of $X$ such that $\cap\left\{B\left(n, x_{n}\right) \mid n \geqslant 1\right\} \neq \varnothing$, then the sequence $\left\langle x_{n}\right\rangle$ must cluster in $X$. 
From the definition of a $w \Delta$-space (presented in Section 2) it is clear that every $w \Delta$-space is a $\beta$-space; in particular, any countably compact space is a $\beta$-space. Hence the space $\omega_{1}$ shows that the existence of a $\beta$-function does not yield covering properties in an ordered space.

The main result in this section is

3.1. Theorem. Let $X$ be a GO space which is hereditarily a $\beta$-space. Then $X$ is (hereditarily) paracompact. in [10]:

That theorem will be proved by contradiction based on the following result

3.2. Theorem. A GO space $X$ is not paracompact if and only if some closed subspace of $X$ is homeomorphic to a stationary subset $\left({ }^{2}\right)$ of a regular $\left({ }^{3}\right)$ uncountable cardinal.

Thus the rest of this section is devoted to provingt hat no stationary set in a regular uncountable cardinal can be hereditarily a $\beta$-space. Once that is established Theorem 3.1 follows from Theorem 3.2.

We begin with a lemma whose proof depends on the next well-known result; short proofs appear in [9] and [4].

3.3. Theorem (Pressing Down Lemma). Let $S$ be a stationary subset of a regular uncountable cardinal $x$ and suppose $f: S \rightarrow \chi$ is a function such that $f(x)<x$ whenever $x \in S-\{0\}$. Then for some $y \in x$ the set $f^{-1}[\{y\}]$ is stationary in $x$.

3.4. LemMA. Let $S$ be a stationary subset of a regular uncountable cardinal $x$. If $S$ is a $\beta$-space, then there is a first countable subspace $T$ of $S$ which is also stationary.

Proof. Let $B$ be a $\beta$-function for $S$. We may assume that each $B(x, n)$ is a convex set. Let $\mathscr{B}(1)=\{B(x, 1) \mid x \in S\}$. Let $S^{d}$ be the set of non-isolated points of the space $S$. Then for each $x \in S^{d}$ there is an ordinal $f(x)<x$ such that $f(x) \in S$ and $[f(x), x] \subset B(x, 1)$. The Pressing Down Lemma 3.3 yields a point $p(1) \in S$ and a stationary set $S_{1} \subset S$ such that $\left.S_{1} \subset\right] p(1), \rightarrow\left[\right.$ and if $x \in S_{1}$ then $[p(1), x] \subset B(x, 1)$. Applying the Pressing Down Lemma recursively, we obtain stationary sets $S_{n}$ and points $p(n) \in S$ such that

1) $\left.S_{n+1} \subset S_{n} \cap\right] p(n+1), x[$;

2) if $x \in S_{n+1}$ then $[p(n+1), x] \subset B(x, n+1)$.

Since $\operatorname{cf}(x)>\omega_{0}$, there is a point $q \in S$ with $q>p(n)$ for cach $n$. Letting $\left.S_{n}^{\prime}=S_{n} \cap\right] q, x[$ we obtain stationary subsets of $S$ such that

3) if $n \geqslant 1$ and $x \in S_{n}^{\prime}$ then $[q, x] \subset B(x, n)$

so that, from the definition of $\beta$-functions,

4) if $s_{n} \in S_{n}^{\prime}$ then the sequence $\left\langle s_{n}\right\rangle$ clusters to a point of $S$.

( $\left.{ }^{2}\right)$ A set $C$ is a $c u b$ in $x$ if $C$ is closed and unbounded ( $=$ cofinal) in $\varkappa$. A set $S \subset \varkappa$ is stationary in $x$ is $S \cap C \neq \varnothing$ for every set $C$ which is a cub in $\%$.

(3) A cardinal $\varkappa$ is regular if $\varkappa$ is not the sum of fewer, smaller carclinals. Equivalently, $x=\mathrm{cf}(x)$ where $\mathrm{cf}(x)$ denotes the cofinality of $x$
Let $T=\left\{x \in S \mid x\right.$ is a limit point of $S$ and $\left.\operatorname{cf}(x)=\omega_{0}\right\}$. Certainly $T$ is a firstcountable subspace of $S$. To show that $T$ is stationary, let $C$ be a cub in $\psi$. Choose $c_{1} \in C$. Since $S_{1}^{\prime}$ and $C$ are cofinal there are points $s_{1} \in S_{1}^{\prime}$ and $c_{2} \in C$ with $c_{1}<s_{1}<c_{2}$. Recursively find points $c_{n} \in C$ and $s_{n} \in S_{n}^{\prime}$ with $c_{n}<s_{n}<c_{n+1}$. By (4), there is a point $p \in S$ to which the sequence $\left\langle s_{n}\right\rangle$ converges. Then $p \in T$. And since $C$ is closed, $p \in C$ so that $T \cap C \neq \varnothing$. Thus $T$ is stationary in $x$, as required.

We can now prove Theorem 3.1. Suppose $X$ is a non-paracompact GO space which is hereditarily a $\beta$-space. There is a stationary subset $S$ in an uncountable regular cardinal $\chi$ which is homeomorphic to a (closed) subspace of $X$. In the light of Lemma 3.4, we may assume that $S$ is first countable and hereditarily a $\beta$-space. Find a subset $T$ of $S$ such that both $T$ and $S-T$ are stationary in $\varkappa$ (see [15]). Since $T$ is a $\beta$-space we can construct (as in Lemma 3.4) stationary subsets $T \supset T_{1} \supset T_{2} \supset \ldots$ with the property that if $t_{n} \in T_{n}$ then $\left\langle t_{n}\right\rangle$ must cluster in $T$. Let $C=\{s \in S \mid$ for some $t_{n} \in T_{n},\left\langle t_{n}\right\rangle$ converges to $\left.s\right\}$. As in Lemma 3.4 the set $C$ is seen to be stationary in $x$ and, since $S$ is first countable, $C$ is closed in $S$. But then $C \cap(S-T) \neq \varnothing$ because $S-T$ is stationary in $\chi$, and that is impossible.

Our next result is an immediate corollary of Theorems 2.3 and 3.1 once it is observed that every $w A$-space is a $\beta$-space. The result will be considerably sharpened by Theorem 4.1 for which it is a lemma.

3.5. Corollary. Let $X$ be a GO space whose every subspace is either a $p$-space, an $M$-space, a wA-space or a quasi-complete space. Then $X$ is (hereditarily) paracompact.

3.6. Remark. There are GO spaces which are hereditarily $\beta$-spaces but not even quasi-complete spaces. The space described in Example 2.4 is one such space.

4. Metrizability of GO spaces with certain hereditary properties. In this section we present our main theorem and its rather lengthy proof.

4.1. Theor:m. Let $X$ be a GO space. The following properties of $X$ are equivalent:

a) $X$ is metrizable;

b) $X$ is hereditarily a p-space (i.e., each subspace of $X$ is a $p$-space);

c) $X$ is hereditarily an $M$-space [13];

d) $X$ is hereditarily a $w \Delta$-space;

e) $X$ is hereditarily quasi-complete.

The implication a) $\rightarrow$ b) is well-known and the equivalence of c), d) and e) follows from Theorem 2.3. That b) implies c) follows from a delicate theorem due independently to van Douwen and van Wouwe [16] asserting that any generalized ordered $p$-space is also an $M$-space. If $X$ satisfies c) then every subspace of $X$ is a paras pert general theory a paracompact $M$-space in the light of 3.1 and it is an easy part of general theory that any paracompact $M$-space is a $p$-space. Thus $\mathrm{c}$ ) implies $\mathrm{b}$ ), and it remain
to show that $\mathrm{b}$ ) implies a). That proof requires a sequence of lemmas. 
4.2. LEMMA. Let $X$ be a linearly ordered set and let $Y \subset X$. Then there are disjoint sets $D, E \subset Y$ such that:

(a) if $p \in X$ and $] p, q[\cap Y$ is infinite for each $q>p$, then for each $q>p$ both ]$p, q[\cap D$ and $] p, q[\cap E$ are infinite;

(b) if $p \in X$ and $] q, p[\cap Y$ is infinite for each $q<p$ then for each $q<p$ both ]$q, p[\cap D$ and $] q, p[\cap E$ are infinite.

Proof. We define $D$ and $E$ recursively. Let $x=|Y|$ and say that a convex set $J$ is $Y$-large if $|Y \cap J| \geqslant 2$. Define $d(x, 0)$ and $e(x, 0)$ to be any two points of $Y$ with $d(x, 0)<e(x, 0)$ and let $\mathscr{I}(0)=\{X\}$. Let $D(0)=\{d(x, 0)\}, E(0)=\{e(x, 0)\}$ and $F(0)=D(0) \cup E(0)$

Suppose $\alpha<x^{+}$and that for each $\beta<\alpha$ we have:

(1) $\mathscr{I}(\beta)$, the set of $Y$-large convex components of $X-\cup\{F(\gamma) \mid \gamma<\beta\}$;

(2) for each $I \in \mathscr{I}(\beta)$ two points $d(I, \beta)<e(I, \beta)$ chosen from $I \cap Y$ in such a way that if $|I \cap Y| \geqslant 6$ then $I \cap] \leftarrow, d(I, \beta)[$ and $I \cap] e(I, \beta), \rightarrow[$ are both $Y$-large;

(3) $D(\beta)=\{d(I, \beta) \mid I \in \mathscr{I}(\beta)\}, E(\beta)=\{e(I, \beta) \mid I \in \mathscr{I}(\beta)\}$ and $F(\beta)=D(\beta) \cup$ $\cup E(\beta)$.

Let $\mathscr{I}(\alpha)=\left\{I \mid I\right.$ is a $Y$-large convex component of $\left.X-\bigcup_{\beta<\alpha} F(\beta)\right\}$. For each $I \in \mathscr{I}(\alpha)$ choose points $d(I, \alpha)<e(I, \alpha)$ in such a way that if $|I \cap Y| \geqslant 6$ then both $I \cap] \leftarrow, d(I, \alpha)[$ and $I \cap] e(I, \alpha), \rightarrow[$ are $Y$-large. Let $D(\alpha)=\{d(I, \alpha) \mid I \in \mathscr{I}(\alpha)\}, E(\alpha)=\{e(I, \alpha) \mid$ $I \in \mathscr{I}(\alpha)\}$ and $F(\alpha)=D(\alpha) \cup E(\alpha)$

This induction terminates in case $\mathscr{I}(\alpha)=\varnothing$; furthermore there must be some $\alpha<x^{+}$such that $\mathscr{I}(\alpha)=\varnothing$ for otherwise $Y$ contains a set of cardinality $x^{+}>|Y|$. Let $\lambda$ be the first ordinal having $\mathscr{T}(\lambda)=\varnothing$. Let $D=\bigcup\{D(\alpha) \mid \alpha<\lambda\}$,

$$
E=\bigcup\{E(\alpha) \mid \alpha<\lambda\}
$$

and $F=D \cup E$

We assert that if $J$ is an open interval which is $Y$-large, then $J$ meets $F=\bigcup\{F(\beta) \mid \beta<\lambda\}$. For otherwise, there is a convex component $I$ of $X-F$ containing $J$. But then $I$ is $Y$-large so $I \in \mathscr{I}(\lambda)=\varnothing$

Now suppose $p \in X$ and $Y \cap] p, q[$ is infinite whenever $q>p$. Fix $q>p$. We claim that $] p, q[$ meets both $D$ and $E$. Since $] p, q[$ is $Y$-large, we know that $] p, q[$ meets $F$. To obtain a contradiction, suppose ]p, $q[\cap E=\varnothing$. Let $\alpha$ be the first ordinal such that $\varnothing \neq F(\alpha) \cap] p, q[=D(\alpha) \cap] p$, q[. Let $I \in \mathscr{I}(\alpha)$ have $d(I, \alpha)$ $\in] p, q[$. Consider $] p, d(I, \alpha)[$; that set is $Y$-large. Let $\beta$ be the first ordinal with $\varnothing \neq] p, d(I, \alpha)[\cap F(\beta)=] p, d(I, \alpha)[\cap D(\beta)$. Necessarily $\beta \geqslant \alpha$. In case $\beta=\alpha$, let $I^{\prime}$ be the member of $\mathscr{I}(\alpha)$ having $\left.d\left(I^{\prime}, \alpha\right) \in\right] p, d(I, \alpha)\left[\right.$. Because $I^{\prime}$ and $I$ are distinct, they are disjoint convex sets so each point of $I^{\prime}$ lies below each point of $I$; in particular $p<d\left(I^{\prime}, \alpha\right)<e\left(I^{\prime}, \alpha\right)<d(I, \alpha)<q$ contrary to $] p, q[\cap E=\varnothing$. Next suppose $\beta>\alpha$. The set $J=] p, d(I, \alpha)[$ is $Y$-large and convex, and $J \cap(\bigcup\{F(\alpha) \mid \alpha<\beta\})$ $=\varnothing$ by minimality of $\beta$. Let $I^{\prime}$ be the convex component of $X-\bigcup F(\alpha)$ which contains $J$. Then $I^{\prime}$ is $Y$-large so that $I^{\prime} \in \mathscr{I}(\beta)$. Because $J \cap D(\beta) \neq \varnothing$ and because distinct members of $I(\beta)$ are disjoint, the point of $J \cap D(\beta)$ must be $d\left(I^{\prime}, \beta\right)$. Then we have $d\left(I^{\prime}, \beta\right)<d(I, \alpha)$. Because $\beta>\alpha, d(I, \alpha) \in \bigcup_{\alpha<\beta} F(\alpha)$. Therefore the convex set $I^{\prime}$ must lie in $] \leftarrow, d(I, \alpha)\left[\right.$. But then $p<d\left(I^{\prime}, \beta\right)<e\left(I^{\prime}, \beta\right)<d(I, \alpha)<q$ showing that $] p, q[\cap E \neq \varnothing$. Hence $] p, q[\cap E=\varnothing$ is impossible.

Next, we show that $] p, q[\cap D \neq \varnothing$. For contradiction, suppose $] p, q[\cap D=\varnothing$ We know that $] p, q[\cap F \neq \varnothing$; let $\alpha$ be the first ordinal having $] p, q[\cap F(\alpha) \neq \varnothing$ Then $] p, q[\cap E(\alpha) \neq \varnothing$. Choose $I \in \mathscr{I}(\alpha)$ having $e(I, \alpha) \in] p, q[$. The set $J=] p, e(I, \alpha)[$ is $Y$-large ... indeed $H \cap Y$ is infinite. Let $\beta$ the first ordinal having $J \cap F(\beta) \neq \varnothing$. Necessarily $\beta \geqslant \alpha$ and $J \cap E(\beta) \neq \varnothing$. Consider the case where $\beta=\alpha$. Let $I^{\prime} \in \mathscr{I}(\alpha)$ have $c^{\prime}\left(I^{\prime}, \alpha\right) \in J$. Since $I^{\prime} \neq I$ are members of $\mathscr{I}(\alpha), I \cap I^{\prime}=\varnothing$; further, since one point of $I^{\prime}$ lies below one point of $I$, each point of $I^{\prime}$ precedes each point of $I$ and we have $p<e\left(I^{\prime}, \alpha\right)<d(I, \alpha)<e(I, \alpha)<q$ showing that ]$p, q\left[\cap D \neq \varnothing\right.$. Therefore $\beta>\alpha$. By minimality of $\beta, J \cap \cup F(\alpha)=\varnothing$. Let $I^{\prime}$ be the convex component of $X-\cup F(\alpha)$ containing $J$. Then $I^{\prime} \cap Y \supset J \cap Y$ is infinite

so $I^{\prime} \in \mathscr{T}(\beta)$. Next observe that $e(I, \alpha) \in \bigcup_{\alpha<\beta} F(\alpha)$ so that the convex set $I^{\prime}$ must be contained in $] \leftarrow, e(I, \alpha)$ [. Since $J \subset I^{\prime}$, it follows that $\left.I^{\prime}=\right] r, e(I, \alpha)$ [ for some point or gap $r<e(I, \alpha)$ or $I^{\prime}=[r, e(I, \alpha)[$ for some $r<e(I, \alpha)$. Now some member or gap $r<e(I, \alpha)$ or $I^{\prime}=\left[r, e(I, \alpha)\left[\right.\right.$. Because $J \subset I^{\prime}$ and distinct members of $\mathscr{I}(\beta)$ are disjoint, it follows that $I^{\prime \prime}=I^{\prime}$. We have, therefore, $e\left(I^{\prime}, \beta\right) \in J$. But, as noted above, the right end point of $I^{\prime}$ must be $e(I, \alpha)$ so that, since $\left|I^{\prime} \cap Y\right| \geqslant \kappa_{0}>6$, the set $K=] e\left(I^{\prime}, \beta\right) e(I, \alpha)[$ must be $Y$-large in the light of the second induction hypothesis. Now consider the $(\beta+1)$ level of the construction. Because $e(I, \alpha)$ is the right end point of $I^{\prime}, K$ is a convex component of $X-\bigcup_{\alpha<(\beta+1)} F(\alpha)$ and $K$ is $Y$-large, so $K \in \mathscr{I}(\beta+1)$. But then there is a point $d(K, \beta+1) \in D(\beta+1)$ and we have $p<e\left(I^{\prime}, \beta\right)<d(K, \beta+1)<e(K, \beta+1)<e(I, \alpha)<q$, showing that $\left.D \cap\right] p, q[\neq \varnothing$, a contradiction.

An analogous argument shows that $D$ and $E$ also satisfy assertion (b) of (4.2)

4.3. LemMA. Let $X$ bo a first countable GO space and let $Z$ be a quasi-developable subspace of $X$. Then there is a sequence $\langle\mathscr{G}(n)\rangle$ of collections of open subsets of $X$ such that if $p \in Z \cap U$, where $U$ is open in $X$, then for some $n, \operatorname{St}(p, \mathscr{G}(n)) \subset U$.

Proof since $Z$ is a quasi-developable GO space it follows from ([12], 5.11) that $Z$ has a $\sigma$. $Z$ has a $\sigma$-disjoint base $\mathscr{B}=U\{D(n) \mid n \geq 1\}$. We may assume that members of $D$ are $Z$-convex base $\mathscr{B}=\bigcup\{\mathscr{B}(n) \mid n \geqslant 1\}$. We may assume that members of member $B$ of $\mathscr{B}$ then $\{z \in Z \mid p<z<q\} \subset B$.

For each $B \in \mathscr{B}$ let $B^{\prime}=\{x \in X \mid$ for some $p, q \in B, p \leqslant x \leqslant q\}$. If the set $B$ is noch that $p(B) \in B^{\prime} \subset[p(B) \rightarrow[$ and $[p(B) \rightarrow[$ is not open in $X$, or (2) there is a point that $p(B) \in B^{\prime} \subset[p(B), \rightarrow[$ and $[p(B), \rightarrow[$ is not open in $X$, or $(2)$ is not open in $X$, 
or both (1) and (2). If case (1) holds, then there is a point $p^{\prime}<p(B)$ such that $\left[p(B), \rightarrow[\cap Z=] p^{\prime}, \rightarrow[\cap Z\right.$ and because $X$ is first countable, we may choose a sequence $\langle p(k, B)\rangle$ of points of $X$ which is strictly increasing and converges to $p(B)$ and for which $] p(k, B), \rightarrow[\cap Z=[p(B), \rightarrow[\cap Z$. And if case (2) holds, there is strictly decreasing sequence $\langle q(k, B)\rangle$ of points of $X$ which converges to $q(B)$ and for which $Z \cap] \leftarrow, q(k, B)[=Z \cap] \leftarrow, q(B)]$.

For each $B \in \mathscr{B}(n)$ define a set $G(k, B)$ by

$$
G(k, B)=\left\{\begin{array}{l}
B^{\prime} \text { if } B^{\prime} \text { is open in } X \\
] p(k, B), q(k, B)[\text { if both cases }(1) \text { and }(2) \text { occur } \\
{[p(B), q(k, B)[\text { if only case (2) occurs }} \\
] p(k, B), q(B)] \text { if only case }(1) \text { occurs }
\end{array}\right.
$$

Let $\mathscr{G}(n, k)=\{G(k, B) \mid B \in \mathscr{B}(n)\}$. Observe that no point of $X$ can belong to more than two distinct members of $\mathscr{G}(n, k)$ and that a point $p \in Z$ can belong to at most one member of $\mathscr{G}(n, k)$.

Now suppose $p \in U \cap Z$ where $U$ is open in $X$. Shrinking $U$ if necessary we may assume $U$ is convex in $X$. Find an integer $n \geqslant 1$ such that some member $B_{0}$ (necessarily unique) of $\mathscr{B}(n)$ satisfies $p \in B_{0} \subset U \cap Z$. Since $U$ is convex, $B_{0}^{\prime} \subset U$. Since $U$ is open, there is a sufficiently large $k$ for which $G\left(k, B_{0}\right) \subset U$. Since no other member of $\mathscr{G}(n, k)$ can contain $p$, we have $\operatorname{St}(p, \mathscr{G}(n, k)) \subset U$ so that any re-indexing of the countable family $\{\mathscr{G}(n, k) \mid n, k \geqslant 1\}$ as a simple sequence is enough to complete the proof.

4.4. Corollary. Let $X$ be a first countable GO space. If $X$ is the union of countably many quasi-developable subspaces, then $X$ is quasi-developable.

Proof. This result follows immediately from 4.3.

We remark that first countability cannot be deleted from the hypothesis of 4.4 ; see Example 2.4. In order to apply 4.4 to our present problem we need:

4.5. Lemma. Let $X$ be a GO space which is hereditarily a p-space. Then $X$ is first countable.

Proof. Fix $q \in X$ and suppose $[q, \rightarrow[$ is not open in $X$. There is a regular cardinal $x$ and an increasing net $\left\{x_{\alpha} \mid \alpha<x\right\}$ of points of $]+\ldots, q[$ which converges to $q$. We may suppose that for each limit ordinal $\lambda<x$ the set $\left\{x_{\alpha} \mid \alpha \leqslant \lambda\right\}$ is closed in $X$. Let $L=\left\{\lambda<x \mid \lambda\right.$ is a limit ordinal and $x_{\lambda}$ is a limit point of $\left.\left\{x_{\alpha} \mid \alpha<\lambda\right\}\right\}$. Let $Y=X-\left\{x_{\lambda} \mid \quad \lambda \in L\right\}$ and let $\bar{X}$ be any ordered compactification of $X$ in the sense of the Introduction. Then $\bar{X}$ is also an ordered compactification of $Y$. Let $\langle\mathscr{P}(n)\rangle$ be a pluming of $Y$ in $\bar{X}$ by collections of convex open subsets of $\bar{X}$. Now suppose $x>\omega_{0}$. Then $S=\bigcap\{\operatorname{St}(q, \not{P}(n)) \mid n \geqslant 1\}$ is a convex subset of $\bar{X}$ which is entirely contained in $Y$. Because $x>\omega_{0}$ and because the ordering of $\bar{X}$ extends the ordering of $Y$, there must be a point $r \in Y$ with $r<q$ and such that the set $T=\{z \in \bar{X} \mid r \leqslant z \leqslant q\}$ is contained in $S$. Note that $T$ is a compact subset of $X$. Let $\beta$ be the first ordinal such that $\left.x_{\beta} \in\right] r, q\left[\right.$. Let $y_{n}=x_{\beta+n}$. Then $\left\langle y_{n}\right\rangle$ is an increasing sequence in the compact set $T$ and yet $\left\langle y^{\prime \prime}\right\rangle$ cannot have a cluster point in $T$, which is impossible. Hence $x=\omega_{0}$.

Similarly, if $\mid$ raw, $q \mid$ is not,open in $X$ then there is a decreasing sequence $\{y(n) \mid n \geqslant 1\}$ in $] q, \rightarrow$ [ which converges to $q$. Combining these two cases we see that $X$ is lirst countable.

Having established those preliminary lemmas, we are ready to begin our proof of Theorem $4.1(\mathrm{~b} \rightarrow \mathrm{a})$. Because of the length of that proof, the reader may find a brief preview to be helpful. In the light of our earlier results, if $X$ is a GO space which is hereditarily a $p$-space, then $X$ is both first countable and hereditarily paracompact. In our proof we create various subspaces $X_{i}(0 \leqslant i \leqslant 4)$ of $X$ whose union is $X$ and such that the set of gaps and pseudogaps of $X_{i}$ has certain points of $X_{i}$ as two-sided limit points. By embedding the GO spaces $X_{i}$ in ordered compactifications, we can then obtain quasi-developments for the spaces $X_{t}$. Then, in view of 4.4, the following general metrization theorem can be applied.

4.6. Theorem [5]. Any quasi-developable paracompact $p$-space is metrizable.

Proof of Theorem 4.1. Let $X_{0}$ be the set of isolated points of $X$. Let $X_{1}$ be the set of (relatively) isolated points of $X-X_{0}$. Both subspaces are quasi-developable; by (4.4) so is $X_{0} \cup X_{1}$.

For $p, q \in X$ define $p \sim q$ if and only if the closed interval between $p$ and $q$, i.e., either $[p, q]$ or $[q, p]$, is a metrizable subspace of $X$. Then $\sim$ is an equivalence relation on $X$ (see below) and we denote the $\sim$ equivalence class to which $x$ belongs by $\operatorname{cls}(x)$. Of course, once (4.1) is proved we will know that $\operatorname{cls}(x)=X$, but until then we can at least assert:

(1) each cls $(x)$ is closed in $X$ and is metrizable;

(2) distinct equivalence classes cannot abutt: indeed if $x<y$ and $\operatorname{cls}(x) \neq \operatorname{cls}(y)$ then there must be uncountably many points $z \in X$ satisfying $x<z<y$ and $z \notin \operatorname{cls}(x) \cup \operatorname{cls}(y)$;

(3) if $|\operatorname{cls}(x)|=1$, then, whenever $p<x<q$, both ],$x$ [ and $] x, q$ [are uncountable;

(4) If $\operatorname{cls}(x)$ has a right end point $b$ in the space $X$ then $b \in \operatorname{cls}(x)$ and for every $q>b$ the set $] b, q$ [ is the union of uncountably many distinct equivalence classes. An analogous statement applies to any left end-point of cls $(x)$ which may belong to $X$.

To justify those assertions let us recall a few fucts from general theory. If a space $X$ is the union of countably many closed, metrizable subspaces then $X$ is semi-stratifiable in the sense of Creede [8]. Furthermore, any semi-stratifiable GO space is metrizable ([12], 5.3). Those two remarks prove that $\sim$ is an equivalence relation and establish (2), (3) and (4) once assertion (1) is proved. Metrizability of each cls $(x)$ follows from Smirnov's metrization theorem since cls $(x)$ is locally metrizable by definition and paracompact in the light of 3.1. To see that $\operatorname{cls}(x)$ is closed in $X$ we note that cls $(x)$ is convex so that if $\operatorname{cls}(x)$ is not closed then it fails to contain one of its end-points, say $p$. But the union of a metric space $M$ and a single point $p$ is metrizable provided 6 - Fundamenta Mathematicae T. CVII/ 1 
$M \cup\{p\}$ is regular and first-countable; therefore by $4.5, \operatorname{cls}(x) \cup\{p\}$ would be metrizable, contradicting maximality of $\operatorname{cls}(x)$. Thus $\operatorname{cls}(x)$ is closed in $X$.

For each $x \in X$ let $I(x)=\operatorname{Int}(\operatorname{cls}(x))$ and let $X_{2}=U\{I(x) \mid x \in X\}$. Because the $I(x)$ 's are pairwise disjoint open metrizable subspaces of $X, X_{2}$ is quasi-developable. By 4.4 , so is $X_{0} \cup X_{1} \cup X_{2}$.

Let $Y=X-\left(X_{0} \cup X_{1} \cup X_{2}\right)$ and let $X_{3}=\{x \in Y|| \operatorname{cls}(x) \mid=1\}$. Apply Lemma 4.2 to the set $Y$ to find disjoint sets $D, E \subset Y$ such that if $p \in X$ is a limit point of $] p, \rightarrow[\cap Y$ then $p$ is also a limit point of $] p, \rightarrow[\cap D$ and of $] p, \rightarrow[\cap E$ (and such that the left-hand analogue of that assertion also holds). Let $X^{\prime}=X-D$ and $X^{\prime \prime}=X-E$. Let $X^{+}$be any ordered compactification of $X$ (in the sense of the Introduction). Then

(5) each $d \in D$ is a limit point in $X$ of $X^{\prime}$ and each $e \in E$ is a limit point in $X$ of $X^{\prime \prime}$.

To verify (5) fix $d \in D$. If $d$ is in the closure of the set $\left(X_{0} \cup X_{1} \cup X_{2}\right) \subset X^{\prime}$ there is nothing to prove, so suppose some open convex neighborhood $U$ of $d$ is disjoint from $\left(X_{0} \cup X_{1} \cup X_{2}\right)$. Then $U \subset Y$. Since $d \notin X_{0}$, one of the sets $\left.] \leftarrow, d\right]$ and $[d, \rightarrow[$ is not open. Assume $] \leftarrow, d]$ is not open in $X$. (The other case is similar.) Then for each $b \in X$ with $b>d$, the set $[d, b[\cap U$ is an infinite subset of $Y$, showing that $d$ is a limit point of $] d, \rightarrow[\cap Y$. Hence $d$ is also a limit point of $] d, \rightarrow\left[\cap E \subset E \subset X^{\prime}\right.$. Thus the first assertion in (5) is established and the second is proved in an analogous way.

It follows from (5) that $X^{+}$is an ordered compactification of both $X^{\prime}$ and $X^{\prime \prime}$ and that each $d \in D$ corresponds to a gap in the space $X^{\prime}$, while each $e \in E$ corresponds to a gap in $X^{\prime \prime}$.

Fix a pluming $\langle\mathscr{P}(n)\rangle$ of $X^{\prime}$ in $X^{+}$. We may assume that $\mathscr{P}(n+1)$ refines $\mathscr{P}(n)$ and that each collection $\mathscr{P}(n)$ consists of convex open subsets of $X^{+}$. Let $X_{3}^{\prime}=X_{3} \cap X^{\prime}=X_{3}-D$ and consider a fixed point $p \in X_{3}^{\prime}$. If there is a point $b \in X$ such that $] p, b[\cap Y=\varnothing$, then $] p, b\left[\subset X_{0} \cup X_{1} \cup X_{2}\right.$ so that $] p, b[$ is seen to be a quasi-developable subspace of $X$. In the light of Theorem 4.6, ]p,b[ must be metrizable whence so is $[p, b]$. But then $\{p, b\} \subset \operatorname{cls}(p)$ which is impossible because $p \in X_{3}^{\prime}$ forces $|\operatorname{cls}(p)|=1$. Therefore $] p, b[\cap Y$ is infinite whenever $b>p$ and, similarly, ]$a, p[\cap Y$ is infinite whenever $a<p$. It follows from Lemma 4.2 that whenever $a<p<b$, each of the sets $] a, p[\cap D] a,, p[\cap E] p,, b[\cap D$ and $] p, b[\cap E$ is infinite.

Since $p \in X_{3}^{\prime}, p \notin X_{0}$ so that exactly one of the following cases applics to $p$ :

(a) $[p, \rightarrow[$ is open in $X$ but $] \leftarrow, p]$ is not;

(b) $] \leftarrow, p]$ is open in $X$ but $[p, \rightarrow[$ is not;

(c) neither $] \leftarrow, p]$ nor $[p, \rightarrow[$ is open in $X$.

Consider case (a). Suppose $p$ is not the left end-point of $X$. Then basic neighborhoods of $p$ in $X$ have the form $\left[p, b[\right.$. Because $\varnothing \neq] a, p[\cap E \subset] a, p\left[\cap X^{\prime}\right.$ for each $a<p, p$ has no immediate predecessor in $X^{\prime}$. Therefore $p$ defines a leftpseudogap $l(p)$ of the space $X^{\prime}$, i.e., a point $l(p)$ of $X^{+}-X^{\prime}$ which is the immediate predecessor of $p$ in the ordering of $X^{+}$. Fix any $b \in X$ with $p<b$; there is a point $d \in D$ with $d \in] p, b\left[\right.$, and $D \subset X^{+}-X^{\prime}$. Because $\bigcap\{\operatorname{St}(p, \mathscr{P}(n)) \mid n \geqslant 1\} \subset X^{\prime}$ there is an $n \geqslant 1$ having $\operatorname{St}(p, \mathscr{P}(n)) \cap\{l(p), d\}=\varnothing$. Because the members of $\mathscr{P}(n)$ are convex subsets of $X^{++}, \operatorname{St}(p, \mathscr{P}(n)) \subset\left\{w \in X^{+} \mid l(p)<w<d\right\}$ showing that, if we write $\mathscr{P}^{\prime}(n)=\left\{P \cap X^{\prime} \mid P \in \mathscr{P}(n)\right\}, \operatorname{St}\left(p, \mathscr{P}^{\prime}(n)\right) \subset[p, b[$. Therefore the sequence $\left\langle\mathscr{P}^{\prime}(n)\right\rangle$ acts as a quasi-development at each point $p \in X_{3}^{\prime}$ described in case (a). (The case where $p$ is the left end-point of $X$ is analogous.)

Case (b) parallels case (a) so we consider points $p \in X_{3}^{\prime}$ as described in case (c) above. Then basic neighborhoods of $p$ in $X^{\prime}$ have the form $] a, b\left[\cap X^{\prime}\right.$ where $a<p<b$ and where $] a, p\left[\cap X^{\prime}\right.$ and $] p, b\left[\cap X^{\prime}\right.$ are infinite sets. Fix $a$ and $b$ with $a<p<b$. Choose points $d_{1}, d_{2} \in D$ with $a<d_{1}<p<d_{2}<b$. Since $d_{1}, d_{2} \in X^{+}-X^{\prime}$ there is an integer $n$ such that $\operatorname{St}(p, \mathscr{P}(n)) \cap\left\{d_{1}, d_{2}\right\}=\varnothing$ so that, with notation as in case (a), $\left.\operatorname{St}\left(p, \mathscr{P}^{\prime}(n)\right) \subset\right] a, b\left[\cap X^{\prime}\right.$. Therefore we see that $X_{3}^{\prime}$ is a quasidevelopable subspace of $X$. A similar argument proves that $X_{3}^{\prime \prime}=X_{3}-E$ is a quasidevelopable subspace of $X$. Because $D \cap E=\varnothing, X_{3}^{\prime} \cup X_{3}^{\prime \prime}=X_{3}$ so that in the light of 4.4 we now see that $\left(X_{0} \cup X_{1} \cup X_{2} \cup X_{3}\right)$ is a quasi-developable subspace of $X$.

Let $X_{4}=X-\left(X_{0} \cup X_{1} \cup X_{2} \cup X_{3}\right)$. If $p \in X_{4}$ then $|\operatorname{cls}(p)| \geqslant 2$ and $p \notin \operatorname{Int}(\operatorname{cls}(p))$ so that $p$ is an end-point of $\operatorname{cls}(p)$. Let $X_{4}^{r}=\left\{p \in X_{4} \mid p=\sup (\operatorname{cls}(p))\right\}$ and $X_{4}^{l}=\left\{p \in X_{4} \mid p=\inf (\operatorname{cls}(p))\right\}$. For each $p \in X_{4}^{r}$ let $L(p)=\{x \in \operatorname{cls}(p) \mid x<p\}$ and note that $] \leftarrow, p]$ is not an open set in $X$.

Define $Z=X-U\left\{L(p) \mid p \in X_{4}^{r}\right\}$. Then:

(6) $X_{4}^{r} \subset Z$;

(7) each $p \in X_{4}^{r}$ defines a left-pseudogap $\hat{p}$ of $Z$, i. e., $[p, \rightarrow[\cap Z$ is open in $Z$ and yet if $a \in Z$ and $a<p$ then $] a, p[\cap Z \neq \varnothing$;

(8) if $p \in Z$ is not the right end-point of $Z$ and if $p<b \in Z$ then $] p, b[\cap Z \neq \varnothing$;

(9) if $p \in X_{4}^{r}$ and $p<z_{0} \in Z$ then either

a) $] p, z_{0}\left[\right.$ contains a point $q \in X_{4}^{r}$ in which case $] p, z_{0}[\cap Z$ contains the left pseudogap $\hat{q}$ associated with $q$ in (7); or

b) $] p, z_{0}\left[\cap X_{4}^{r}=\varnothing\right.$, in which case $] p, z_{0}[\cap Z$ must contain a gap or pseudogap of the space $Z$.

One can justify the alternatives described in (9) as follows. By maximality of the set $\operatorname{cls}(p)$, for each $b$ in $] p, z_{0}[$, the interval $] p, b[$ cannot be metrizable and therefore cannot even be quasi-developable. Therefore, for each $b$ in $] p, z_{0}\left[, X_{4} \cap\right] p, b[\neq \varnothing$. Hence if we assume that $] p, z_{0}\left[\cap X_{4}^{r}=\varnothing\right.$, then $] p, b\left[\cap X_{4}^{l} \neq \varnothing\right.$ whenever $b \in] p, z_{0}$ [ and we may choose distinct $q^{\prime}, q^{\prime \prime}$ in $X_{4}^{l}$ having $p<q^{\prime}<q^{\prime \prime}<z_{0}$. Consider the set cls $\left(q^{\prime}\right)$. If the supremum of $\operatorname{cls}\left(q^{\prime}\right)$ is a gap of the set $X$ then it is also a gap of the set $Z$ and lies in $] p, z_{0}$ [. And if $\sup \left(\operatorname{cls}\left(q^{\prime}\right)\right)$ is a point $s \in X$ then, because ]$p, z_{0}\left[\cap X_{4}^{r}=\varnothing, s \in \operatorname{Int}\left(\operatorname{cls}\left(q^{\prime}\right)\right)\right.$ and $\left.] \leftarrow, s\right]$ is open in $X$. By maximality of the set $\operatorname{cls}\left(q^{\prime}\right), s$ cannot have an immediate successor in $X$. Hence $s$ defines a right pseudogap of $X$, and also a right pseudogap of $Z$, which belongs to the interval $] p, z_{0}[$. Thus assertion (9) is established. Assertion (9) can be rephrased as: 
$(9)^{\prime}$ if $p \in X_{4}^{r}$ and $p<z_{0} \in Z$ then $] p, z_{0}[$ contains a gap or pseudogap of the space $Z$.

Now consider $\bar{Z}$, any ordered compactification of $Z$, and let $\langle\mathscr{R}(n)\rangle$ be a pluming of $Z$ in $\hat{Z}$ by open convex subsets of $\hat{Z}$ such that $\mathscr{R}(n+1)$ refines $\mathscr{R}(n)$. Fix $p \in X_{4}^{r}$ and $b \in X$ with $b>p$, and consider the open set $[p, b[\cap Z$. Let $\hat{p}$ denote the left pseudogap determined by $p$, i.e., $\hat{p}$ is the immediate predecessor of $p$ in $\hat{Z}$, and let $u$ be any gap or pseudogap of $Z$ lying in $[p, b[\cap Z$. Then $\{\hat{p}, u\} \subset \hat{Z}-Z$ so that for some $n,\{\hat{p}, u\} \cap \operatorname{St}(p, \mathscr{R}(n))=\varnothing$. Because the members of $\mathscr{R}(n)$ are convex, $Z \cap \operatorname{St}(p, \mathscr{R}(n)) \subset] \hat{p}, u\left[\cap Z \subset\left[p, b\left[\cap Z\right.\right.\right.$ so that $\left\{X_{4}^{r} \cap \operatorname{St}(p, \mathscr{R}(n)) \mid n \geqslant 1\right\}$ is seen to be a neighborhood base for $p$ in $X_{4}^{r}$. Thus $X_{4}^{r}$ is a quasi-developable subspace of $X$.

An analogous argument proves that $X_{4}^{l}$ is quasi-developable. Since $X=\bigcup_{i=0}^{3} X_{i} \cup$ $\cup X_{4}^{r} \cup X_{4}^{l}, X$ is quasi-developable in the light of 4.4 ; hence $X$ is metrizable.

\section{References}

[1] A. V. Arhangel'skǐr, On a class of spaces containing all metric spaces and all locally bicompact spaces, Sov. Math. Dokl. 4 (1963), pp. 751-754.

[2] - On hereditary properties, Gen. Top. Appl. 3 (1973), pp. 39-46.

[3] C. E. Aull, Topological spaces with a $\sigma$-point finite base, Proc. Amer. Math. Soc. 29 (1971), pp. 411-416.

[4] J. Baumgartner and K. Prikry, Singular cardinals and the Generalized Continulum Hypothesis, Amer. Math. Monthly 84 (1977), pp. 108-113.

[5] H. R. Bennett, A note on the metrizability of $M$-spaces, Proc. Japan Acad. 45 (1969), pp. 6-9.

[6] - On quasi-developable spaces, Gen. Top. Appl. 1 (1971), pp. 253-262.

[7] C. R. Borges, On metrizability of topological spaces, Canad. J. Math. 20 (1968), pp. 795-804.

[8] G. Creede, Concerning semistratifiable spaces, Pacific J. Math. 32 (1970), pp. 47-54.

[9] E. van Douwen and D. Lutzer, On the classification of stationary sets, Michigan Math. J. 26 (1979), pp. 47-64.

[10] R. Engelking and D. Lutzer, Paracompactness in ordered spaces, Fund. Math. 94 (1977), pp. $49-58$.

[11] R. Hodel, Moore spaces and w4-spaces, Pacific J. Math. 38 (1971), pp. 641-652.

[12] D. Lutzer, On generalized ordered spaces, Dissertationes Math. 89, 1971, pp. 36.

[13] K. Morita, Products of normal spaces with metric spaces, Math. Ann. 154 (1964), pp. 365-382.

[14] L. Steen, $A$ direct proof that a linearly ordered space is hereditarily collectionwise normal, Proc. Amer. Math. Soc. 24 (1970), pp. 727-728.

[15] R. Solovay, On the solution of a problem of Fodor and Hajnal, Proc. Summer Inst. on Axiomatic Set Theory, A. M. S. Proc. Symp. Pure Math. 13.

[16] J. van Wouwe, On generalized ordered p-and $M$-spaces, Wiskundig Sem. Report \#52, Free University, Amsterdam, August, 1976. 